\title{
Construction of an Uncertainty to Maximize the Gain at Multiple Frequencies
}

\author{
Bálint Patartics, Peter Seiler and Bálint Vanek
}

\begin{abstract}
This paper considers the construction of worstcase perturbations for uncertain systems. The uncertain system is modeled as an interconnection of a linear time-invariant (LTI) system and a norm-bounded LTI uncertainty. The worst-case gain (measured in the $H_{\infty}$ norm) for the uncertain system can be assessed via skewed- $\mu$ analysis. The standard approach is to compute upper and lower bounds for the worst-case gain on a frequency grid. A worst-case LTI perturbation is then constructed to maximize the gain at a single frequency. This perturbation can be used within a high fidelity nonlinear simulation to further explore system robustness. A drawback of this existing approach is that the worst-case perturbation constructed at a single frequency may not necessarily induce poor time-domain performance. It is beneficial to construct a perturbation that maximizes the gain at multiple frequency points, e.g. where the system is most sensitive or where disturbances have large frequency content. This paper provides an algorithm to construct a single perturbation which causes the uncertain system to achieve its largest possible gain at multiple chosen frequency points. This is achieved by interpolating through worst-case samples at the individual frequencies using the boundary Nevanlinna-Pick interpolation. Simple numerical examples are provided to demonstrate the proposed approach.
\end{abstract}

\section{INTRODUCTION}

The worst-case gain [1] is a useful metric for analyzing the robustness of uncertain systems. This is defined as the maximum $H_{\infty}$ norm of the uncertain system over the set of allowable uncertainties. This performance measure is often used in analysis and occasionally in control synthesis e.g. [2], [3]. The worst-case gain can be assessed by a skewed structured singular value (skewed- $\mu$ ) calculation. This problem is known to be NP-hard [4] and therefore efficient techniques for obtaining upper and lower bounds have been developed.

The upper bound calculation is turned into a convex optimization problem using D-G scales [5], [6]. The lower bound involves heuristics for constructing "bad" uncertainty samples, i.e. those that achieve large gain for the uncertain system. A common heuristic is the skewed- $\mu$ power iteration [7]. This yields a complex (structured) matrix which causes the uncertain system to achieve the worst-case gain lower bound at a given frequency. A corresponding linear time-invariant (LTI) uncertainty sample is constructed by interpolating the complex matrix at the given frequency. This

B. Patartics and B. Vanek are with Hungarian Academy of Sciences Institute for Computer Science and Control, H-1111 Budapest, Kende u. 13-17., Hungary patartics.balintasztaki.mta.hu and vanek@sztaki.mta. hu

P. Seiler is with the Aerospace Engineering and Mechanics Department, University of Minnesota, 107 Akerman Hall, 110 Union St. SE, Minneapolis MN 55455, USA seile017@umn. edu

The research leading to these results is part of the FLEXOP and the FLIPASED projects. These projects have received funding from the Horizon 2020 research and innovation programme of the European Union under grant agreement No. 636307 and 815058 respectively. single frequency interpolation is always possible using a stable LTI system with norm bounded by that of the complex matrix [8, Theorem 9.1]. These bounds can be computed with the Robust Control toolbox (wcgain) [9] or the Systems Modeling, Analysis and Control (SMAC) toolbox [10].

This paper presents a new method to construct worst-case LTI uncertainty samples. The proposed algorithm maximizes the gain of the uncertain system at multiple (given) frequency points. This is in contrast to the existing method that computes an uncertainty to maximize the worst-case gain lower bound at a single frequency (often the peak frequency). The proposed method uses complex matrix uncertainty samples computed by the worst-case gain lower bound power iteration at multiple frequencies. Next, the boundary Nevanlinna-Pick (BNP) method [11] is used to create a stable, norm-bounded LTI uncertainty that interpolates this collection of matrix samples.

This uncertainty sample is useful for further time and frequency domain analyses. E.g. it can be incorporated in a higher fidelity nonlinear simulation, especially for systems with multiple resonance frequencies such as hard disk drives [12], and flexible aircraft [13]. In addition, it can be useful as part of control design methods that require bad samples of dynamic uncertainty [2]. Selecting uncertainty samples which provide large gain at multiple frequencies could improve the convergence speed of such synthesis methods. The MATLAB implementation of the proposed algorithm along with the examples presented in this paper can be downloaded from [14].

The most closely related work is [15] which considers sample construction for randomly sampling an uncertainty set. The Nevanlinna-Pick theorem is employed to sample unit $H_{\infty}$ norm systems. The approach involves randomly chosen frequencies and data that are interpolated while constraining the resulting interpolant to be stable and norm bounded by one. The construction in [15] is similar to the one presented in this paper. The main difference is that random uncertainty samples are created in [15] while our method aims to maximize gain at multiple frequencies. It is also noted that the method proposed in this paper might be used to construct lower bounds on the worst-case $\mathrm{H}_{2}$ norm of an uncertain system. This will be the subject of future work. If successful, it would provide lower bounds on the robust $\mathrm{H}_{2}$ norm to complement the upper bounds in [16], [17].

The rest of the paper has the following outline. Section II details the problem statement. This includes the classical worst-case uncertainty construction method and the notation used in the paper. In Section III, the BNP interpolation is described. The worst-case uncertainty construction with interpolation at multiple frequencies is given in Section IV. 


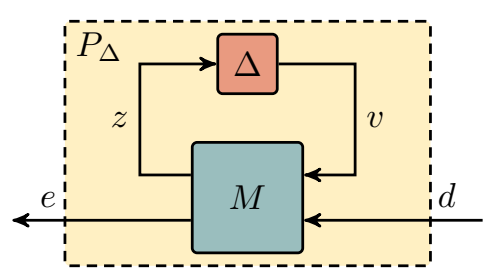

Fig. 1. Uncertain system interconnection.

Numerical examples are provided in Section $\mathrm{V}$ and conclusions are drawn in Section VI.

\section{PRoBlem STATEMENT}

\section{A. Notation}

Let $X \in \mathbb{C}^{r_{X} \times c_{X}}$ and $Y \in \mathbb{C}^{r_{Y} \times c_{Y}}$ be matrices with $c_{Y}<r_{X}$ and $r_{Y}<c_{X}$. Partition $X$ such that

$$
X=\left[\begin{array}{ll}
X_{11} & X_{12} \\
X_{21} & X_{22}
\end{array}\right]
$$

and $X_{11}$ is $r_{Y} \times c_{Y}$. The upper linear fractional transformation (LFT) is $\mathcal{F}_{U}(X, Y)=X_{22}+X_{21} Y\left(I-X_{11} Y\right)^{-1} X_{12}$, where $I$ is the identity matrix of appropriate dimensions.

Denote the conjugate transpose of $Y$ by $Y^{*}$ and it's largest singular value by $\bar{\sigma}(Y)$. If $X$ and $Y$ are the same size, then $X>Y$ (or $<, \geq, \leq$ ) denotes element wise inequality. For $X=X^{*}$ and $Y=Y^{*}$ the expression $X \succ Y$ means that $X-Y$ is positive definite. The symbols $\prec, \succeq, \preceq$ are to be understood accordingly. The block diagonal concatenation of $Y_{1}, \ldots, Y_{n}$ is denoted by $\operatorname{diag}\left(Y_{1}, \ldots, Y_{n}\right)$.

\section{B. Worst-Case Gain}

Consider the block diagram in Fig. 1. This is an interconnection of a stable, multi-input multi-output (MIMO) LTI system $M(s)$ and a structured dynamic uncertainty $\Delta(s)$. The uncertain system is

$$
P_{\Delta}(s)=\mathcal{F}_{U}(M(s), \Delta(s)) .
$$

Define the set of structured and unit norm bounded dynamic uncertainties as

$$
\begin{array}{r}
\Delta:=\left\{\operatorname{diag}\left(\Delta_{1}(s), \ldots, \Delta_{N_{\mathrm{b}}}(s)\right), \Delta_{i}(s)\right. \text { is LTI, } \\
\left.\left\|\Delta_{i}(s)\right\|_{\infty} \leq 1, i=1, \ldots, N_{\mathrm{b}}\right\} .
\end{array}
$$

Notice, that $\Delta(s) \in \Delta$ implies $\|\Delta(s)\|_{\infty} \leq 1$. The block $\Delta_{i}(s)$ can be MIMO and has dimensions $r_{i} \times c_{i}$.

The worst-case gain of the uncertain system is the maximum induced $L_{2}$ gain (i.e. $H_{\infty}$ norm) of $P_{\Delta}(s)$ over the set of allowable uncertainties:

$$
\hat{\gamma}:=\max _{\Delta \in \Delta}\left\|\mathcal{F}_{U}(M(s), \Delta(s))\right\|_{\infty} .
$$

The interconnection in Fig. 1 is assumed to be robustly stable, i.e. $P_{\Delta}(s)$ is stable for all $\Delta(s) \in \mathbb{\Delta}$. This implies $\hat{\gamma}<\infty$. The worst-case gain defined in (2) is equivalent to calculating the peak gain of the system frequency by frequency $\hat{\gamma}=\max _{\omega} \gamma(\omega)$, where $\gamma(\omega)$ is the peak gain of $P_{\Delta}(s)$ at $\omega$, i.e.

$$
\gamma(\omega)=\max _{Q \in \mathbb{Q}} \bar{\sigma}\left(\mathcal{F}_{U}(M(j \omega), Q)\right) .
$$

Here, the set of block structured, unit norm bounded complex uncertainties is

$$
\begin{array}{r}
\mathbb{Q}:=\left\{\operatorname{diag}\left(Q_{1}, \ldots, Q_{N_{\mathrm{b}}}\right), Q_{i} \in \mathbb{C}^{r_{i} \times c_{i}}, \bar{\sigma}\left(Q_{i}\right)=1,\right. \\
\left.\operatorname{rank}\left(Q_{i}\right)=1, i=1, \ldots, N_{\mathrm{b}}\right\} .
\end{array}
$$

Note that $\mathcal{Q} \in \mathbb{Q}$ implies $\bar{\sigma}(\mathcal{Q})=1$. In addition, note that it is sufficient to restrict the complex matrices to be rank one as in the definition of $\mathbb{Q}[5]$, [8].

A standard approach is to approximate $\hat{\gamma}$ by performing the calculation in (3) on a finite frequency grid. Specifically, a sufficiently dense grid $\left\{\omega_{k}\right\}_{k=1}^{N_{\omega}}$ is chosen. At each frequency, $\gamma\left(\omega_{k}\right)$ is the result of a skewed- $\mu$ analysis which for many cases is known to be NP-hard [4]. Instead, lower bounds $\left\{L_{k}\right\}_{k=1}^{N_{\omega}}$ and upper bounds $\left\{U_{k}\right\}_{k=1}^{N_{\omega}}$ are computed such that $L_{k} \leq \gamma\left(\omega_{k}\right) \leq U_{k}, k=1, \ldots, N_{\omega}$.

The upper bounds are computed via semi-definite programming involving scaling matrices to account for the structure of $\Delta[6]$. The lower bounds are obtained employing a skewed- $\mu$ power iteration [5], [7]. The power iteration at $\omega_{k}$ yields $\mathcal{Q}_{k} \in \mathbb{Q}$ such that

$$
\bar{\sigma}\left(\mathcal{F}_{U}\left(M\left(j \omega_{k}\right), \mathcal{Q}_{k}\right)\right)=L_{k} .
$$

Finally, the worst-case gain is bounded by $\max _{k} L_{k} \leq \hat{\gamma} \leq$ $\max _{k} U_{k}$. This computation is performed in the MATLAB function wcgain [9]. Similar calculations are performed by the MATLAB-Simulink Systems Modeling, Analysis and Control (SMAC) toolbox [10].

\section{Single Frequency Interpolation}

The existing approach to worst-case uncertainty construction is given in the proof of Theorem 9.1 (small gain theorem) in [8]. Suppose that the lower bound power iteration at $\omega_{0}$ yields the lower bound $L_{0}$ and worst-case perturbation $\mathcal{Q}_{0}=\operatorname{diag}\left(Q_{1}, \ldots, Q_{N_{\mathrm{b}}}\right) \in \mathbb{Q}$. The goal is to find an LTI uncertainty which interpolates $\mathcal{Q}_{0}$ at the single frequency $\omega_{0}$. The uncertainty must be stable, unit norm bounded and have the correct block structure, i.e. we want to find $\Delta_{\mathrm{s}}(s) \in \mathbb{\Delta}$ (the 's' subscript refers to single peak). This uncertainty drives the gain of the uncertain system to the power iteration lower bound at $\omega_{0}$, in other words it satisfies $\bar{\sigma}\left(\mathcal{F}_{U}\left(M\left(j \omega_{0}\right), \Delta_{\mathrm{s}}\left(j \omega_{0}\right)\right)\right)=L_{0}$.

Each block $Q_{i} \in \mathbb{C}^{r_{i} \times c_{i}}$ of $\mathcal{Q}_{0}$ is rank-one with unit maximum singular value, i.e. each block can be expressed as $Q_{i}=u v^{*}$ for some vectors $\|u\|_{2}=\|v\|_{2}=1$. Let $u_{l} \in \mathbb{C}$ denote the $l^{\text {th }}$ element of the vector $u$. It is possible to find a SISO transfer function $\hat{u}_{l}(s)$ such that $\hat{u}_{l}\left(j \omega_{0}\right)=u_{l}$ and $\left\|\hat{u}_{l}(s)\right\|_{\infty} \leq\left|u_{l}\right|$. This SISO interpolation can be performed with a constant or first-order transfer function $\hat{u}_{l}(s)$ as described in the proof of Theorem 2 in Appendix A. Each entry of $u$ can be interpolated to obtain an $r_{i} \times 1$ stable system $\hat{u}(s)$ with $\|\hat{u}(s)\|_{\infty} \leq 1$. Similarly, each entry of $v^{*}$ can be interpolated to obtain a $1 \times c_{i}$, stable system $\hat{v}(s)$ with $\|\hat{v}(s)\|_{\infty} \leq 1$. Finally, the block $\Delta_{i}(s)=\hat{u}(s) \hat{v}(s)$ is stable and satisfies $\Delta_{i}\left(j \omega_{0}\right)=Q_{i}$ and $\left\|\Delta_{i}\right\|_{\infty} \leq 1$. As a result, $\Delta_{\mathrm{s}}(s)=\operatorname{diag}\left(\Delta_{1}(s), \ldots, \Delta_{N_{\mathrm{b}}}(s)\right) \in \Delta$ interpolates $\mathcal{Q}_{0}$ at frequency $\omega_{0}$.

The wcgain function computes the worst-case uncertainty using this method. In the implementation, a bandpass filter is added to the uncertainty. This band-pass filter is omitted from the discussions in this paper. 


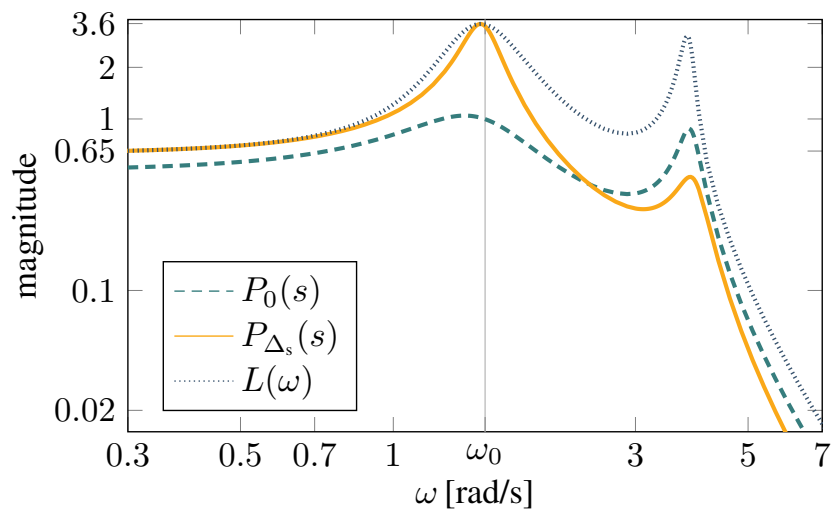

Fig. 2. The gain of the uncertain system $P_{\Delta}(s)$ for different values of the uncertainty.

One entry of $u$ can be normalized in each block of $Q_{i}=$ $u v^{*}$. Specifically, $\|u\|_{2}=1$ implies $u$ has at least one nonzero entry, say $u_{l} \neq 0$. Then $Q_{i}=\tilde{u} \tilde{v}^{*}$ where $\tilde{u}:=u / u_{l}$ and $\tilde{v}:=u_{l} v$. This normalizes the $l^{\text {th }}$ entry, i.e. $\tilde{u}_{l}=1$. Therefore, if $\mathcal{Q}_{0} \in \mathbb{C}^{r_{\mathcal{Q}} \times c_{\mathcal{Q}}}$ then in general $\Delta(s)$ has $r_{\mathcal{Q}}+$ $c_{\mathcal{Q}}-N_{b}$ states. The number of states can be less if there are real numbers in the rank-one decomposition of the blocks of $\mathcal{Q}_{0}$. The most notable situations when that happens is when $\omega_{0}=0$ or $\omega_{0}=\infty$. In those cases, $M\left(j \omega_{0}\right)$ is a real matrix therefore the power iteration results in a real $\mathcal{Q}_{0}$.

Typically, this method is used to interpolate the matrix $\mathcal{Q}_{0}$ that achieves the maximal lower bound. Therefore, $\Delta_{\mathrm{S}}(s)$ achieves the largest gain found by the power iteration over all frequencies in the grid. However, the gain $\bar{\sigma}\left(\mathcal{F}_{U}\left(M(j \omega), \Delta_{\mathrm{s}}(j \omega)\right)\right)$ may not be large at other frequencies $\left(\omega \neq \omega_{0}\right)$.

A simple flexible control example is presented to demonstrate this. The open-loop system is $G_{\text {flex }}(s)=G_{1}(s) G_{2}(s)$ consisting of two uncertain flexible modes

$$
\begin{aligned}
& G_{1}(s)=\frac{1}{s^{2}+0.8 s+1}\left(1+0.3 \Delta_{1}(s)\right) \\
& G_{2}(s)=\frac{16}{s^{2}+0.4 s+16}\left(1+0.3 \Delta_{2}(s)\right),
\end{aligned}
$$

where $\left\|\Delta_{1}(s)\right\|_{\infty} \leq 1$, and $\left\|\Delta_{2}(s)\right\|_{\infty} \leq 1$. The uncertain system of interest is the closed loop $P_{\Delta}(s)=\frac{G_{\text {flex }}(s)}{1+G_{\text {flex }}(s)}$ with $\Delta(s)=\operatorname{diag}\left(\Delta_{1}(s), \Delta_{2}(s)\right)$. Because the $P_{\Delta}(s)$ contains two flexible modes, we know a priory that it is sensitive to excitation at (at least) two frequencies. Fig. 2 confirms this by showing the gain of the nominal system $P_{0}(s)$ as well as the worst-case gain lower bound $L(\omega)$. The maximum of the lower bound occurs at $\omega_{0}=1.52 \mathrm{rad} / \mathrm{s}$. The skewed$\mu$ power iteration yields $\mathcal{Q}_{0}=\operatorname{diag}\left(Q_{1}, Q_{2}\right)$ where $Q_{1}=$ $Q_{2}=0.34-0.94 j$. The uncertainty constructed with the classical interpolation method (see Appendix A) is

$$
\Delta_{1}(s)=\Delta_{2}(s)=-\frac{s-2.111}{s+2.111} .
$$

Thus, $\Delta_{\mathrm{s}}(s)=\operatorname{diag}\left(\Delta_{1}(s), \Delta_{2}(s)\right)$. As depicted in Fig. 2, this uncertainty sample drives the magnitude of $P_{\Delta_{\mathrm{s}}}(s)$ to its maximum at $\omega_{0}$. However, the gain of $P_{\Delta_{s}}(s)$ at the second peak is less than the gain of the nominal system $P_{0}(s)$.

\section{Objective: Multiple Frequency Interpolation}

The example from the previous section motivates the need to move beyond the typical single frequency interpolation in worst-case gain analysis. This section provides a concrete formulation for the multiple frequency interpolation problem addressed by this paper.

Assume the following are given: a robustly stable uncertain system in (1) with $\Delta(s) \in \Delta$, a collection of frequencies $\left\{\omega_{k}\right\}_{k=1}^{N_{\omega}}$, worst-case gain lower bounds $\left\{L_{k}\right\}_{k=1}^{N_{\omega}}$, and complex matrices $\left\{\mathcal{Q}_{k}\right\}_{k=1}^{N_{\omega}} \subset \mathbb{Q}$ for which (4) hold. Find $\Delta_{\mathrm{m}}(s) \in \triangle$ such that

$$
\bar{\sigma}\left(\mathcal{F}_{U}\left(M\left(j \omega_{k}\right), \Delta_{\mathrm{m}}\left(j \omega_{k}\right)\right)\right)=L_{k}
$$

for each $k=1, \ldots, N_{\omega}$. The 'm' subscript stands for multiple peak.

To achieve this, we propose an interpolation of $\left\{\mathcal{Q}_{k}\right\}_{k=1}^{N_{\omega}}$ at frequencies $\left\{\omega_{k}\right\}_{k=1}^{N_{\omega}}$. The interpolant $\Delta_{\mathrm{m}}(s) \in \Delta$ is obtained by the application of the BNP interpolation method which is detailed in Section III. The uncertainty construction is explained in Section IV.

\section{BNP INTERPOLATION}

This section briefly reviews the BNP interpolation method. The algorithm is given in Example 21.3.1 and Corollary 21.4.2 in [11] and is repeated here for ease of reference.

Theorem 1: Let $\left\{w_{k}\right\}_{k=1}^{N_{w}}$ be given, distinct numbers on the imaginary axis. In addition, let $\left\{u_{k}\right\}_{k=1}^{N_{w}} \subset \mathbb{C}^{q}$ and $\left\{v_{k}\right\}_{k=1}^{N_{w}} \subset \mathbb{C}^{q}$ be given unit-length vectors. The following statements hold for any such interpolation data.

1) There exist non-negative real numbers $\left\{\rho_{k}\right\}_{k=1}^{N_{w}}$ such that the boundary Pick matrix $H(\rho) \in \mathbb{C}^{N}{ }^{N} \times N_{w}$ defined by

$$
H_{i k}(\rho)= \begin{cases}\frac{v_{i}^{*} v_{k}-u_{i}^{*} u_{k}}{w_{i}^{*}+w_{k}}, & i \neq k \\ \rho_{k}, & i=k,\end{cases}
$$

satisfies $H(\rho) \succ 0$.

2) Assume $H(\rho) \succ 0$ and define

$$
\begin{aligned}
C_{0-} & :=\left[\begin{array}{lll}
v_{1} & \ldots & v_{N_{w}}
\end{array}\right] \in \mathbb{C}^{q \times N_{w}}, \\
C_{0+} & :=\left[\begin{array}{lll}
u_{1} & \ldots & u_{N_{w}}
\end{array}\right] \in \mathbb{C}^{q \times N_{w}}, \\
A_{0} & :=\operatorname{diag}\left(w_{1}, \ldots, w_{N_{w}}\right) \in \mathbb{C}^{N_{w} \times N_{w}}, \\
\Theta(s) & :=I+\left[\begin{array}{l}
C_{0+} \\
C_{0-}
\end{array}\right]\left(s I-A_{0}\right)^{-1} H(\rho)^{-1}\left[\begin{array}{c}
-C_{0+} \\
C_{0-}
\end{array}\right]^{*} .
\end{aligned}
$$

Let $G(s)$ be any $q \times q$ rational function analytic on the closed right-half plane with $\|G(s)\|_{\infty} \leq 1$. Define

$$
\begin{aligned}
\Delta(s):= & \left(\Theta_{11}(s) G(s)+\Theta_{12}(s)\right) \cdot \\
& \left(\Theta_{21}(s) G(s)+\Theta_{22}(s)\right)^{-1},
\end{aligned}
$$

where $\Theta(s):=\left[\begin{array}{cc}\Theta_{11}(s) & \Theta_{12}(s) \\ \Theta_{21}(s) & \Theta_{22}(s)\end{array}\right]$ is partitioned into $q \times q$ blocks. Then $\Delta(s)$ is analytic on the closed right-half plane and $\|\Delta(s)\|_{\infty} \leq 1$. Moreover, $\Delta\left(w_{k}\right) v_{k}=u_{k}$, $u_{k}^{*} \Delta\left(w_{k}\right)=v_{k}^{*}$, and $-u_{k}^{*} \Delta^{\prime}\left(w_{k}\right) v_{k}=\rho_{k}$ for $k=$ $1, \ldots, N_{w}^{\dagger}$.

Proof: See [11].

${ }^{\dagger}$ The ()$^{\prime}$ superscript denotes derivative with respect to the independent variable. 


\section{Multiple Frequency Interpolation}

The main contribution is summarized in this section.

\section{A. Application of the BNP Interpolation}

The basic BNP interpolation result in Theorem 1 is applied but with several modifications. First, the theorem assumes that the interpolation vectors are both of the same dimension. Zero-padding is used if the dimensions are not equal. For example, assume $\left\{u_{k}\right\}_{k=1}^{N_{w}}$ and $\left\{v_{k}\right\}_{k=1}^{N_{w}}$ have dimensions $r$ and $c$, respectively, with $r>c$. In this case, the vectors $\left\{v_{k}\right\}_{k=1}^{N_{w}}$ are augmented with $r-c$ zeros so that $\tilde{v}_{k}^{*}=\left[\begin{array}{ll}v_{k}^{*} & 0\end{array}\right]$. The resulting interpolant is $\tilde{\Delta}(s)=\left[\begin{array}{ll}\Delta(s) & \Delta_{0}(s)\end{array}\right]$ where $\tilde{\Delta}(s)$ satisfies the interpolation conditions with $\left\{u_{k}\right\}_{k=1}^{N_{w}}$ and $\left\{\tilde{v}_{k}\right\}_{k=1}^{N_{w}}$. Then $\Delta(s)$ satisfies the interpolation conditions with $\left\{u_{k}\right\}_{k=1}^{N_{w}}$ and $\left\{v_{k}\right\}_{k=1}^{N_{w}}$. Similarly, $\left\{u_{k}\right\}_{k=1}^{N_{w}}$ are augmented with zeros when $r<c$.

Next, the interpolant provided by Theorem 1 is not necessarily a real system, i.e. its coefficients in transfer function or state-space form can be complex. A system with real coefficients is obtained by interpolating the given data as well as its complex conjugate. Specifically, assume the given interpolation data is $\left\{u_{k}\right\}_{k=1}^{N_{\omega}},\left\{v_{k}\right\}_{k=1}^{N_{\omega}}$, and a collection of non-negative frequencies $\left\{\omega_{k}\right\}_{k=1}^{N_{\omega}}$. The interpolation from Theorem 1 is then constructed with the data

$$
\begin{array}{ccccccc}
w_{k}: & -j \omega_{N_{\omega}} & \ldots & -j \omega_{1} & j \omega_{1} & \ldots & j \omega_{N_{\omega}} \\
u_{k}: & u_{N_{\omega}}^{*} & \ldots & u_{1}^{*} & u_{1} & \ldots & u_{N_{\omega}}
\end{array}
$$

If zero is among the frequency points then it is not duplicated. This makes $N_{w}=2 N_{\omega}$ or $N_{w}=2 N_{\omega}-1$ if the zero frequency is included. It can be shown that interpolating such conjugate data yields an interpolant $\Delta(s)$ with real coefficients.

Finally, Theorem 1 provides a set of interpolants depending on the choices for the non-negative numbers $\left\{\rho_{k}\right\}_{k=1}^{N_{w}}$ and rational function $G(s)$. An all-pass $\Delta(s)$ is obtained if $G(s)$ is chosen to be all-pass [18]. For simplicity, we always pick $G(s)=I$. In addition, the interpolant satisfies $-u_{k}^{*} \Delta^{\prime}\left(w_{k}\right) v_{k}=\rho_{k}$ and hence smaller values of $\left\{\rho_{k}\right\}_{k=1}^{N_{w}}$ are related to smoother interpolants. The following optimization is formulated to obtain a "smooth" interpolant. Let $R:=$ $\operatorname{diag}\left(\rho_{1}, \ldots, \rho_{N_{w}}\right)$ be the main diagonal of the boundary Pick matrix $H(\rho)$ defined in (6). Define $H_{0}:=H(\rho)-R$. The following optimization is solved to obtain $\left\{\rho_{k}\right\}_{k=1}^{N_{w}}$ :

$$
\begin{aligned}
\min & \hat{\rho}+\operatorname{trace}(R) \\
& \text { subject to: } \\
& \hat{\rho} \geq R \geq 0 \\
& \kappa I \succeq H_{0}+R \succeq \frac{1}{\kappa} I .
\end{aligned}
$$

This objective function combines a bound on the peak value and the sum of $\left\{\rho_{k}\right\}_{k=1}^{N_{w}}$. The solution of this optimization leads to a "smooth" interpolant. The two terms are weighted equally in this formulation but alternative weightings could be used. The optimization also includes a upper bound constraint on the condition number $\kappa$ of the boundary Pick matrix $H(\rho)$. This constraint improves the conditioning of

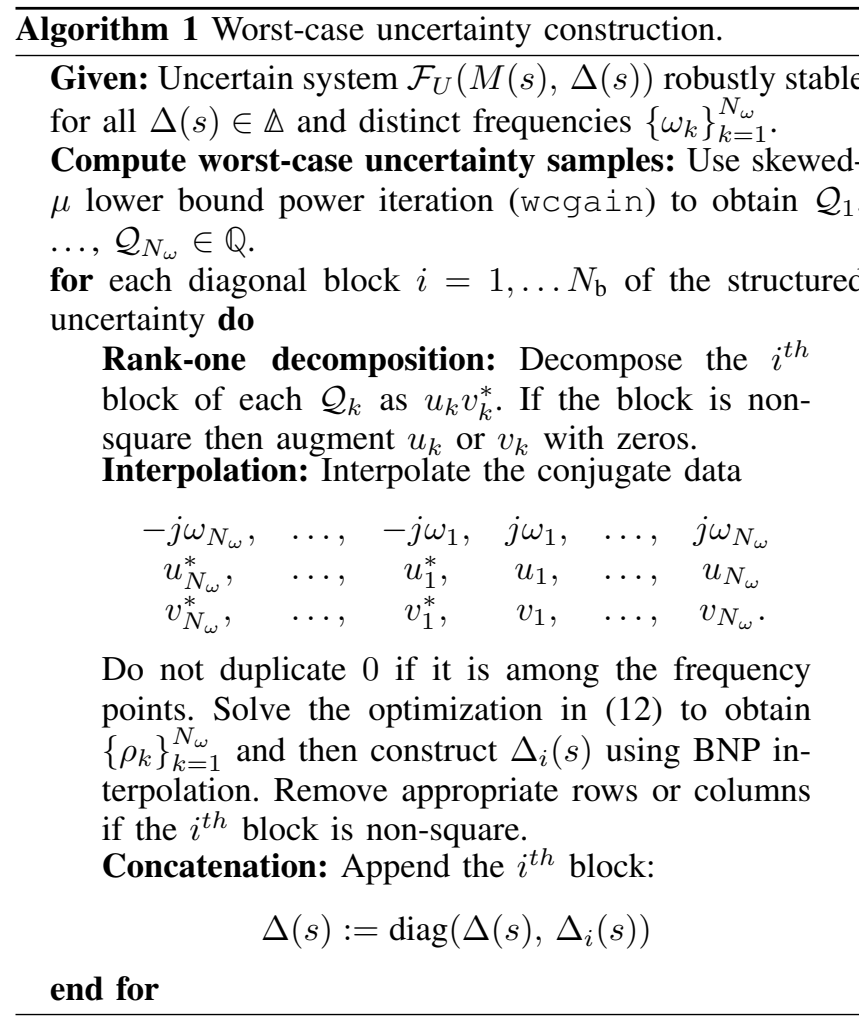

the matrix inversion $H(\rho)^{-1}$ that appears in (10). The condition number bound is selected as $\kappa=10^{4}$.

\section{B. Worst-Case Uncertainty Construction}

Given are the robustly stable uncertain system in (1) along with a block structure $\Delta$, and distinct frequencies $\left\{\omega_{k}\right\}_{k=1}^{N_{\omega}}$. We want to find the worst case uncertainty as defined in Section II-D. To achieve this, we first compute uncertainty samples at $\left\{\omega_{k}\right\}_{k=1}^{N_{\omega}}$ and use the BNP interpolation block by block to obtain the interpolant $\Delta_{\mathrm{m}}(s) \in \triangle$. The method is summarized in Algorithm 1.

The worst-case uncertainty samples $\left\{\mathcal{Q}_{k}\right\}_{k=1}^{N_{\omega}}$ are complex matrices computed on the frequency grid $\left\{\omega_{k}\right\}_{k=1}^{N_{\omega}}$ using the existing worst-case gain lower bound power iteration [7]. The uncertainty $\Delta_{\mathrm{m}}(s) \in \Delta$ is the result of interpolation between these matrices. The uncertainty samples have block diagonal structure, i.e. $\mathcal{Q}_{k}=\operatorname{diag}\left(Q_{1, k}, \ldots, Q_{N_{b}, k}\right) \in \mathbb{Q}$ has block-diagonal structure. Thus, the interpolation procedure in Theorem 1 and Section IV-A is repeated for each block separately. Let $Q_{i, k}$ denote the $i^{\text {th }}$ block of sample $\mathcal{Q}_{k}$. For this block, compute a rank-one decomposition at each frequency $Q_{i, k}=u_{i, k} v_{i, k}^{*}, k=1, \ldots, N_{\omega}$. If $Q_{i, k}$ is not square, add zeros at the end of $u_{i, k}$ or $v_{i, k}$, whichever has fewer elements, so that they have the same size. Form the input interpolation data to the BNP procedure as in (11). Apply BNP interpolation as described in Sections III and IVA. This procedure interpolates through the rank-one blocks of the uncertainty sample. The $i^{\text {th }}$ block of the resulting $\Delta_{i}(s)$ will interpolate $\left\{u_{i, k}\right\}_{k=1}^{N_{w}}$ and $\left\{v_{i, k}\right\}_{k=1}^{N_{w}}$ but can, in general, be a full rank matrix at the interpolation frequencies.

In Theorem $1, \Delta_{i}(s)$ interpolates $u_{i, k}$ and $v_{i, k}$ in the sense that $\Delta_{i}\left(j \omega_{k}\right) v_{i, k}=u_{i, k}$ for $k=$ 
$1, \ldots, N_{\omega}$. At $\omega_{k}$, the lower bound power iteration yields $\mathcal{Q}_{k}=\operatorname{diag}\left(u_{1, k} v_{1, k}^{*}, \ldots, u_{N_{b}, k} v_{N_{b}, k}^{*}\right)$ for which $\bar{\sigma}\left(\mathcal{F}_{U}\left(M\left(j \omega_{k}\right), \mathcal{Q}_{k}\right)\right)=L_{k}$. That means that there exist unit vectors $u_{p, k}$ and $v_{p, k}$ such that $\mathcal{F}_{U}\left(M\left(j \omega_{k}\right), \mathcal{Q}_{k}\right) u_{p, k}=$ $L_{k} v_{p, k}$. Let $u_{k}^{*}:=\left[\begin{array}{lll}u_{1, k}^{*} & \cdots & u_{N_{b}, k}^{*}\end{array}\right]$ and $v_{k}^{*}:=$ $\left[\begin{array}{lll}v_{1, k}^{*} & \cdots & v_{N_{b}, k}^{*}\end{array}\right]$. Then the interpolation means that $\Delta_{\mathrm{m}}\left(j \omega_{k}\right) v_{k}=u_{k}$. This is sufficient since the uncertainty satisfies the equations

$$
\begin{aligned}
M\left(j \omega_{k}\right)\left[\begin{array}{c}
u_{k} \\
u_{p, k}
\end{array}\right] & =\left[\begin{array}{c}
v_{k} \\
L_{k} v_{p, k}
\end{array}\right] \\
\Delta_{\mathrm{m}}\left(j \omega_{k}\right) v_{k} & =u_{k}
\end{aligned}
$$

which implies (5).

Note that because of the definition of $A_{0}$ in (9) and our choice of $G(s)=I$, the state order of the interpolant in Theorem 1 is $N_{w}$. Since the interpolation is repeated for every block the number of states in $\Delta_{\mathrm{m}}(s)$ is $N_{w} N_{\mathrm{b}}=$ $2 N_{\omega} N_{\mathrm{b}}$ (or $\left(2 N_{\omega}-1\right) N_{\mathrm{b}}$ ). Thus, if $N_{\omega}=1$ this method generally provides a lower dimensional uncertainty sample than the classical solution in Section II-C.

\section{EXAMPLES}

In this section, two examples are presented for the application of Algorithm 1. The first is a vibration example consisting of a SISO system which has two dominant frequencies. The second demonstrates the algorithm in a more general situation with a MIMO system and a more complicated block structure.

\section{A. Vibration control example}

This section revisits the example introduced in Section IIC. The nominal system has two resonance frequencies at $\omega_{1}=1.52 \mathrm{rad} / \mathrm{s}$, and $\omega_{2}=3.83 \mathrm{rad} / \mathrm{s}$ as illustrated in Fig. 2. The proposed algorithm is used to compute the worstcase uncertainty $\Delta_{\mathrm{m}}(s) \in \Delta$ which maximizes the gain of $P_{\Delta}(s)$ at $\omega_{1}$ and $\omega_{2}$ simultaneously. At $\omega_{2}$, the power iteration yields $Q_{1}=Q_{2}=0.79+0.62 j$. The uncertainty sample at $\omega_{1}$ is provided in Section II-C. Interpolating the uncertainty samples at both frequencies yields $\Delta_{\mathrm{m}}(s)=$ $\operatorname{diag}\left(\Delta_{1}(s), \Delta_{2}(s)\right)$ where

$$
\begin{aligned}
& \Delta_{1}(s)=\Delta_{2}(s)= \\
& \quad \frac{(s-269.3)(s-22.33)\left(s^{2}-1.58 s+6.627\right)}{(s+269.3)(s+22.33)\left(s^{2}+1.58 s+6.627\right)} .
\end{aligned}
$$

As shown in Fig. 3, $P_{\Delta}(s)$ with this uncertainty achieves the worst-case gain lower bound at both $\omega_{1}$ and $\omega_{2}$.

Consider the response of $P_{\Delta}(s)$ to the excitation at $0 \mathrm{rad} / \mathrm{s}$, $10 \omega_{2}$, and the resonance frequencies $\omega_{1}$ and $\omega_{2}$. Specifically, Fig. 4 shows the output of $P_{\Delta}(s)$ to the input $u(t)=$ $\theta(t)\left[1+\sin \left(\omega_{1} t\right)-\cos \left(\omega_{2} t\right)+\sin \left(10 \omega_{2} t\right)\right]$ where $\theta(t)$ is the unit step function. Fig. 4 confirms the frequency domain results by displaying additional harmonics in the response of $P_{\Delta_{\mathrm{m}}}(s)$ compared to $P_{0}(s)$ and $P_{\Delta_{\mathrm{s}}}(s)$. It is worth noting that the choice of the interpolant impacts the $\mathrm{H}_{2}$ norm as follows: $\left\|P_{0}(s)\right\|_{H_{2}}=0.75,\left\|P_{\Delta_{\mathrm{s}}}(s)\right\|_{H_{2}}=1.30$, while $\left\|P_{\Delta_{\mathrm{m}}}(s)\right\|_{H_{2}}=1.45$. The multiple-frequency interpolant $\Delta_{\mathrm{m}}(s)$ increases the $H_{2}$ norm in this example but a general relationship remains as future work.

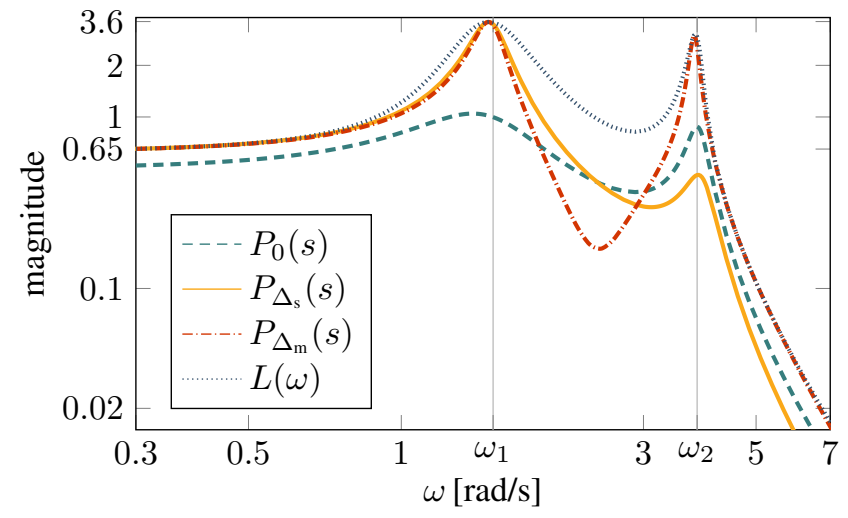

Fig. 3. Worst-case gain lower bound $L$ for the uncertain system $P_{\Delta}(s)$ along gain achieved with different uncertainty values.

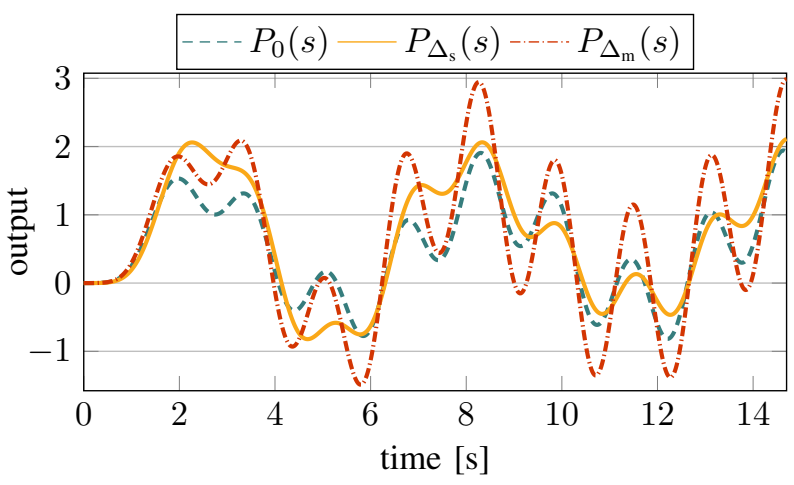

Fig. 4. Response of the uncertain system $P_{\Delta}(s)$ for different values of the uncertainty to an excitation at frequencies $0 \mathrm{rad} / \mathrm{s}, \omega_{1}, \omega_{2}, 10 \omega_{2}$.

\section{B. General example}

Consider an uncertain system $P_{\Delta}(s)=\mathcal{F}_{U}(M(s), \Delta(s))$ where $M(s)$ has 10 states, 13 outputs, and 12 inputs. The uncertainty is $\Delta(s)=\operatorname{diag}\left(\Delta_{1}(s), \Delta_{2}(s), \Delta_{3}(s)\right)$, where $\Delta_{1}(s)$ is $4 \times 2, \Delta_{2}(s)$ is $2 \times 2, \Delta_{3}(s)$ is $3 \times 5$. The uncertainty is assumed to be unit-norm bounded, $\|\Delta(s)\|_{\infty} \leq 1$. The system $M(s)$ is constructed such that $P_{\Delta}(s)$ is robustly stable. For the numerical data see [14].

The goal is to compute the uncertainty sample $\Delta_{\mathrm{m}}(s) \in \mathbb{\Delta}$ which maximizes the gain of $P_{\Delta}(s)$ at the frequency points $\omega_{1}=0.1 \mathrm{rad} / \mathrm{s}, \omega_{2}=2 \mathrm{rad} / \mathrm{s}, \omega_{3}=22 \mathrm{rad} / \mathrm{s}$, and $\omega_{4}=$ $200 \mathrm{rad} / \mathrm{s}$. Since in this case $N_{\omega}=4$ with nonzero frequencies and $N_{\mathrm{b}}=3$, the proposed algorithm yields a $\Delta_{\mathrm{m}}(s)$ with $2 N_{\omega} N_{\mathrm{b}}=24$ states. The gain of the sample is one at every frequency, i.e. $\bar{\sigma}\left(\Delta_{\mathrm{m}}(j \omega)\right)=1 \forall \omega$. Fig. 5 compares the largest singular values of the nominal $P_{0}(s)$ and $P_{\Delta_{\mathrm{m}}}(s)$. As expected, the curve with $\Delta_{\mathrm{m}}(s)$ has maximum singular value which achieves the the worst-case gain lower bound curve at the frequencies $\omega_{1}, \ldots, \omega_{4}$.

The classical construction described in Section II-C gives $\Delta_{\mathrm{s}}(s) \in \mathbb{\Delta}$. This interpolant maximizes the gain of $P_{\Delta_{\mathrm{s}}}(s)$ only at the frequency $\omega_{2}$ where the worst-case gain lower bound achieves its peak. This single frequency interpolation yields a $\Delta_{\mathrm{s}}(s)$ with 15 states. If the BNP method is applied only to this single (maximal) frequency then the an interpolant can be obtained with only $2 N_{\mathrm{b}}=6$ states. 


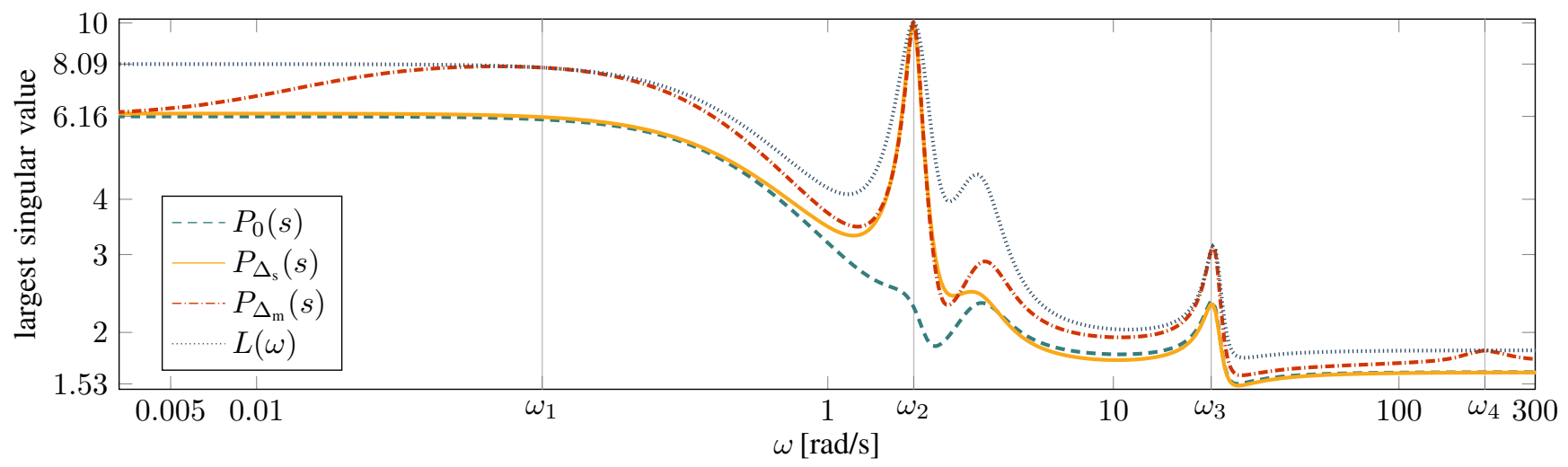

Fig. 5. Worst-case gain lower bound $L$ for the uncertain system $P_{\Delta}(s)$ along with the gain achieved with different uncertainty values.

This indicates that the BNP interpolation has some benefit (in terms of state-order) even when applied to the standard single-frequency interpolation.

\section{CONCLUSION}

An uncertainty construction method is provided that maximizes the gain of an uncertain system at multiple (given) frequency points. This is achieved employing worst-case gain lower bound power iteration to obtain bad uncertainty samples. The samples are interpolated using BNP interpolation. Two examples were presented to demonstrate the application of the theoretical results in the paper. In both cases, the resulting uncertainty produced worse frequency domain behavior than the classical worst-case uncertainty.

\section{APPENDIX}

\section{A. Single Frequency SISO interpolation}

Theorem 2: A finite frequency $\omega_{0}$ and a complex number $\delta_{0} \in \mathbb{C}$ are given. There exists a stable SISO LTI system $\hat{\delta}(s)$ that interpolates the data $\hat{\delta}\left(j \omega_{0}\right)=\delta_{0}$, and whose magnitude $|\hat{\delta}(j \omega)| \leq\left|\delta_{0}\right| \forall \omega$.

Proof: First, consider the fact that a system of the form $T(s)=\frac{s-\beta}{s+\beta}$ with $\beta>0$ is stable and has unit magnitude over all frequencies. Moreover, the phase of $T(s)$ goes from $180^{\circ}$ to $0^{\circ}$ with increasing frequency. Similarly, $-T(s)$ is stable with unit magnitude and has phase that goes from $360^{\circ}$ to $180^{\circ}$. Thus, a transfer function of the form $\pm c \frac{s-\beta}{s+\beta}$ with $c>0$ can achieve any desired phase and magnitude at any frequency.

If $\delta_{0} \in \mathbb{R}$ then select $\hat{\delta}(s)=\delta_{0}$. If $\operatorname{Im}\left(\delta_{0}\right) \neq 0$, then $\delta_{0}= \pm c e^{j \phi}$ for some $c>0$ and $\phi \in\left(0^{\circ}, 180^{\circ}\right)$. Note that since $\beta>0$, the phase of $T(s)$ is

$$
\begin{aligned}
\arg T(j \omega) & =\arg \frac{\omega+j \beta}{\omega-j \beta}=\arg (\omega+j \beta)-\arg (\omega-j \beta) \\
& =\tan ^{-1}\left(\frac{\beta}{\omega}\right)-\tan ^{-1}\left(\frac{-\beta}{\omega}\right)=2 \tan ^{-1}\left(\frac{\beta}{\omega}\right) .
\end{aligned}
$$

To achieve the desired phase $\phi$ at $\omega_{0}$, select $\beta=\omega_{0} \tan \left(\frac{\phi}{2}\right)$ so that $T\left(j \omega_{0}\right)=e^{j \phi}$. Finally, define $\hat{\delta}(s)= \pm\left|\delta_{0}\right| \frac{s-\beta}{s+\beta}$ with the appropriate sign. Then, $\hat{\delta}(s)$ is stable with pole $-\beta$ $(\beta>0), \hat{\delta}\left(j \omega_{0}\right)=\delta_{0}$, and $\|\hat{\delta}(s)\|_{\infty}=\left|\delta_{0}\right|$.

\section{REFERENCES}

[1] A. Packard, G. Balas, R. Liu, and J.-Y. Shin, "Results on worst-case performance assessment," in American Control Conference, vol. 4, 2000, pp. 2425-2427.

[2] R. S. d. S. de Aguiar, P. Apkarian, and D. Noll, "Structured robust control against mixed uncertainty," IEEE Transactions on Control Systems Technology, 2017.

[3] B. Patartics, B. Vanek, and P. Seiler, "Structured robust synthesis with parameter-dependent D-scales," in American Control Conference, 2019, pp. 1800-1805.

[4] R. P. Braatz, P. M. Young, J. C. Doyle, and M. Morari, "Computational complexity of/spl mu/calculation," IEEE Transactions on Automatic Control, vol. 39, no. 5, pp. 1000-1002, 1994.

[5] A. Packard and J. Doyle, "The complex structured singular value," Automatica, vol. 29, no. 1, pp. 71-109, 1993.

[6] R. Holland, P. Young, and C. Zhu, "Development of a skew $\mu$ upper bound," International Journal of Robust and Nonlinear Control, vol. 15 , no. 18 , pp. 905-921, 2005.

[7] _ - "Development of a skew $\mu$ lower bound," International Journal of Robust and Nonlinear Control, vol. 15, no. 11, pp. 495-506, 2005.

[8] K. Zhou, J. C. Doyle, and K. Glover, Robust and optimal control. Prentice hall New Jersey, 1996, vol. 40.

[9] G. Balas, R. Chiang, A. Packard, and M. Safonov, "Robust control toolbox users guide r2014b," 2016.

[10] C. Roos, "Systems modeling, analysis and control (smac) toolbox: An insight into the robustness analysis library," in IEEE Conference on Computer Aided Control System Design, 2013, pp. 176-181.

[11] J. A. Ball, I. Gohberg, and L. Rodman, Interpolation of Rational Matrix Functions. Springer Basel AG, 1990.

[12] Y. Li, R. Horowitz, and R. Evans, "Vibration control of a PZT actuated suspension dual-stage servo system using a PZT sensor," IEEE Transactions on Magnetics, vol. 39, no. 2, pp. 932-937, 2003.

[13] B. Takarics, B. Patartics, T. Luspay, B. Vanek, C. Roessler, J. Bartasevicius, S. J. Koeberle, M. Hornung, D. Teubl, M. Pusch, M. Wüstenhagen, T. M. Kier, G. Looye, P. Bauer, Y. M. Meddaikar, S. Waitman, and A. Marcos, "Active flutter mitigation testing on the flexop demonstrator aircraft."

[14] B. Patartics, P. Seiler, and B. Vanek. Github repository of the matlab implementation of the algorithm. [Online]. Available: https://github.com/Patartics-Balint/wcunc

[15] C. M. Lagoa, X. Li, M. C. Mazzaro, and M. Sznaier, "Sampling random transfer functions," in Probabilistic and Randomized Methods for Design under Uncertainty. Springer, 2006, pp. 331-363.

[16] M. Sznaier, T. Amishima, P. A. Parrilo, and J. Tierno, "A convex approach to robust $\mathcal{H}_{2}$ performance analysis," Automatica, vol. 38, no. 6, pp. 957-966, 2002.

[17] F. Paganini, "Convex methods for robust $\mathrm{H}_{2}$ analysis of continuoustime systems," IEEE Transactions on Automatic Control, vol. 44, no. 2, pp. 239-252, 1999.

[18] J. C. Doyle, B. A. Francis, and A. R. Tannenbaum, Feedback control theory. Courier Corporation, 1990. 